\title{
Implementing a Regional Analgesia Service for Hip Fracture Patients
}

\author{
J Parmar, A Hassan, N Bedforth \\ Queens Medical Centre, Nottingham University Hospitals NHS Trust
}

\section{Background}

Hip fracture causes severe pain and optimising pain control in this group of patients can be challenging. Simple analgesia and opioids are commonly used however they are relatively ineffective for dynamic pain and opioid use in the older population group is associated with significant side effects [1].

Regional analgesia in the form of a fascia iliaca compartment block (FICB) or femoral nerve block prior to surgery results in dynamic analgesia and a reduction in opioid requirements [2]. A Cochrane review has shown that regional blockade reduces pain on movement within 30 minutes after block placement [3].

The National Hip Fracture Database (NHFD) showed our hospital pre-theatre block rate was approximately $8 \%$ compared to $50 \%$ nationally [4]. This highlights an area of improvement for this service.

\section{Aims}

To improve regional analgesia delivery to patients admitted with hip fractures

Improve dynamic pain scores post-regional block

Reduce opioid requirements

\section{Methods}

The emergency department (ED) was identified as a suitable location for patients to receive a FICB, providing early analgesia.

Identifying a permanent group of staff to perform these blocks is vital for sustainability. Our ED is staffed by Advanced Care Practitioners (ACPs) 24 hourly; as they are a stable workforce within the department they should be able to provide FICBs for these patients. As a result the ACPs have received training and supervision of these blocks. A guideline has been produced to assist them further.

For ward patients, we have implemented a 'Block Grab Bag' containing essential equipment for anaesthetists to provide regional analgesia on the ward. Specialist trauma nurses track patients and request blocks from an anaesthetist if the patient has not received one.

\section{Results}

We have collected data on 32 anaesthetic delivered femoral nerve blocks reviewing pre- and post-block:

- Static and dynamic pain scores (Figures 1 \& 2)

- Opioid consumption

We defined dynamic pain as a $15^{\circ} \mathrm{leg}$ raise.

The mean (SD) opioid consumption for each patient pre-block was 10.5 (11.6) mg compared to 1.8 (3.1) $\mathrm{mg}$ post-block up till the time of surgery.

The NHFD run chart (Figure 3 ) shows an increase in our monthly block rate since the start of this project in September 2018, with the block rate in June 2019 at 25\%.

\section{References}

I. Trads, M. and Pedersen, P.U. (2015), “Constipation and defecation pattern the first 30 days after hip fracture", International Journal of Nursing Practice, Wiley Online Library, Vol. 21 No. 5, pp. 598 604.

2. Steenberg, J. and Møller,A.M. (2018), "Systematic review of the effects of fascia iliaca compartment block on hip fracture patients before operation", British Journal of Anaesthesia, Elsevier,Vol. I 20 No. 6, pp. 1368-1380.

3. Guay, J., Parker, M.J., Griffiths, R. and Kopp, S. (2017), "Peripheral nerve blocks for hip fractures”, Cochrane Database of Systematic Reviews, John Wiley \& Sons, Ltd, No. 5.

4. Royal College of Physicians. (2019), "National Hip Fracture Database”, Part of the Falls and Fragility Fracture Audit Programme.

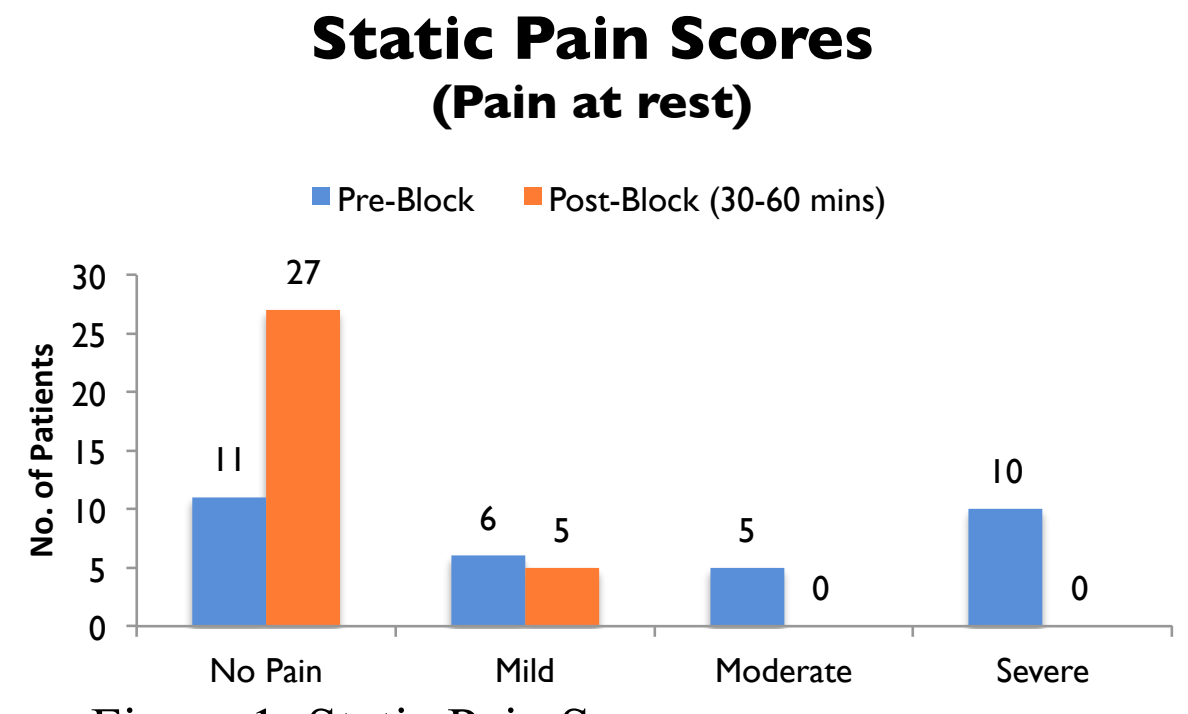

Figure 1: Static Pain Scores

Dynamic Pain Scores

(Pain on attempting $15^{\circ}$ leg raise)

- Pre-Block - Post-Block (30-60 mins)

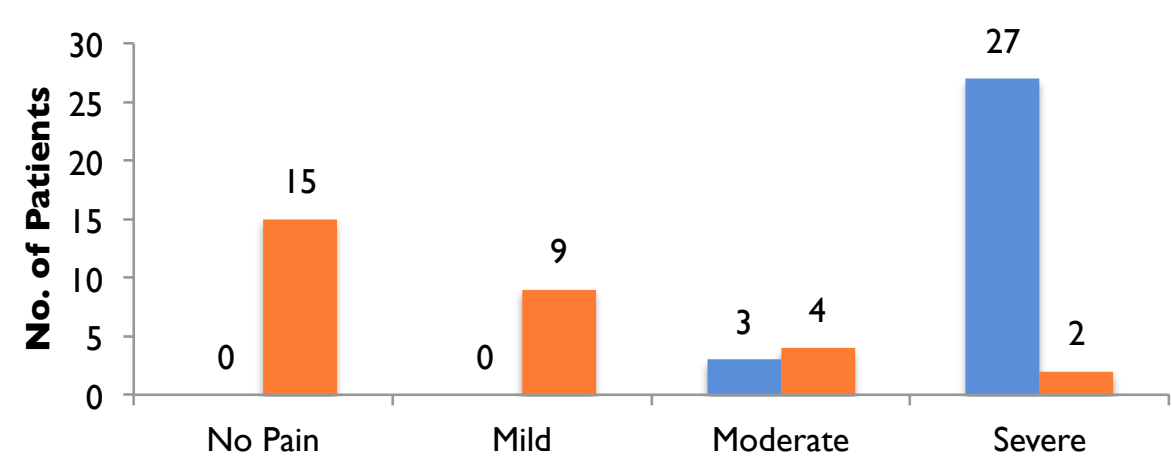

Figure 2: Dynamic Pain Scores

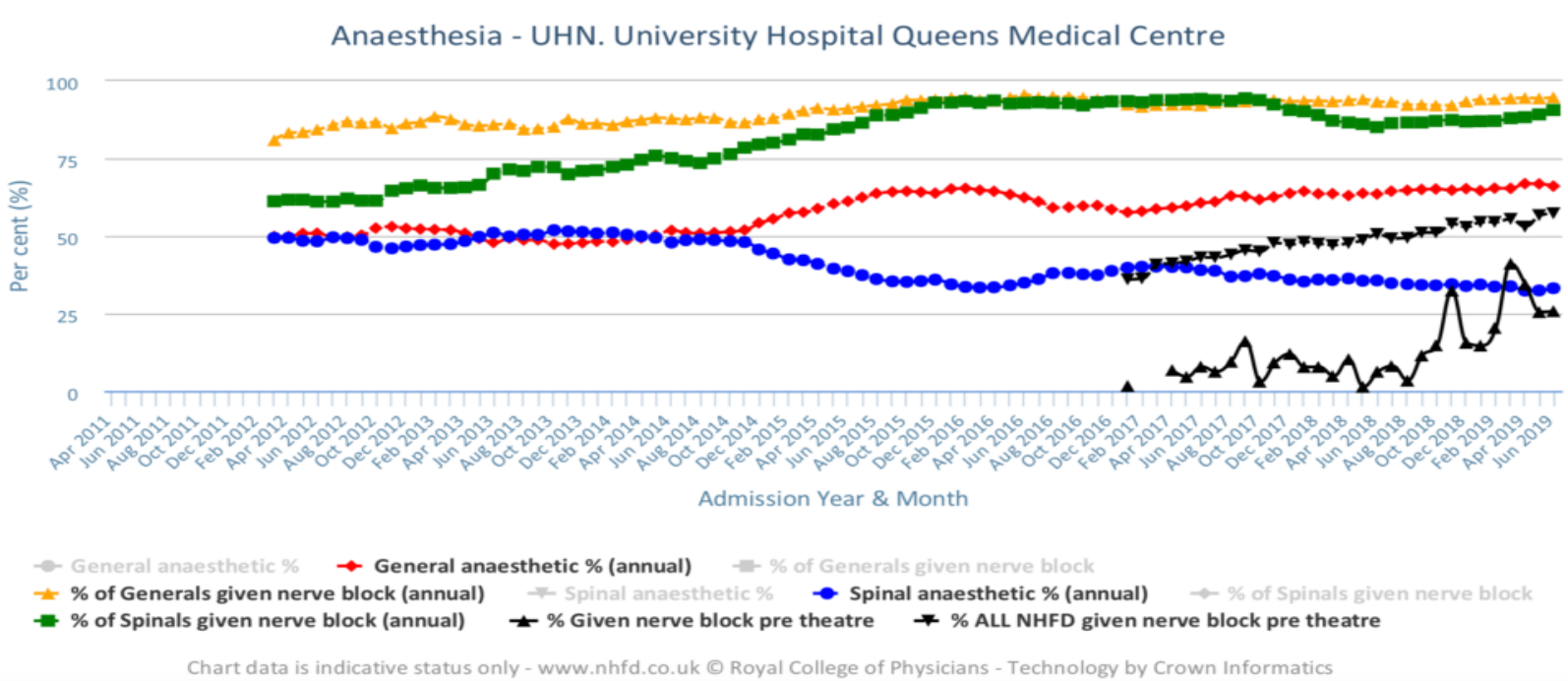

Figure 3: NHFD overall pre-theatre nerve block rate [4]

\section{Conclusions}

We have implemented a pre-operative block pathway for hip fracture patients and demonstrated efficacy in a small sample group. Our total pre-theatre nerve block rate has risen. We plan to support ED ACPs further with block performance with the development of a visual cognitive aid and implementing a 'Block Equipment Trolley' within ED.

\section{Contact:}

For further information please email jainaparmar@doctors.org.uk 